\title{
Antimicrobianos en neonatología. Parte II: Recomendaciones para la dilución y administración de antimicrobianos utilizados en el recién nacido
}

\author{
Antimicrobials in neonatology. Part II: Recommendations for the dilution and \\ administration of antimicrobials in the newborn
}

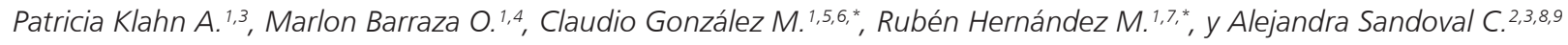

\author{
${ }^{1}$ Químico Farmacéutico. \\ ${ }^{2}$ Médico Infectólogo Pediátrico. \\ ${ }^{3}$ Hospital Carlos Van Buren. Valparaíso, Chile. \\ ${ }^{4}$ Hospital Dr. Luis Calvo Mackenna. Santiago, Chile. \\ ${ }^{5}$ Hospital Exequiel González Cortez. Santiago, Chile. \\ ${ }^{6}$ Comité Consultivo Antimicrobianos SOCHINF. \\ ${ }^{7}$ Hospital Clínico San Borja Arriarán. Santiago, Chile. \\ ${ }^{8}$ Universidad de Valparaíso. Valparaíso, Chile. \\ ${ }^{9}$ Comité Consultivo infecciones Neonatales SOCHINF.
}

*Los autores declaran reciente cambio de afiliación a Complejo Asistencial Barros Luco y Hospital Exequiel González Cortés, respectivamente.

Los autores declaran no tener conflicto de interés.

Este trabajo no posee fuentes de financiamiento.

Recibido / Aceptado: 15 de junio de 2021

\section{Resumen}

Los antimicrobianos corresponden al grupo de medicamentos más utilizados en Unidades de Cuidados Intensivos Neonatales; no obstante, su uso ha sido asociado a constantes errores de medicación en la práctica clínica. Paradojalmente, aún no existe consenso en torno a la administración adecuada de estos medicamentos y existen importantes brechas de conocimiento en torno a los procesos de dosificación, administración y manipulación de antimicrobianos en esta población. Con el fin de mejorar el uso de antimicrobianos, disminuir errores y optimizar los resultados clínicos en el recién nacido, la presente revisión tiene por objetivo entregar recomendaciones y servir de guía para la correcta preparación de aquellos antimicrobianos de mayor relevancia en neonatología.

Palabras clave: recién nacidos; antimicrobianos; dosificación; administración; dilución.

\begin{abstract}
Antimicrobials are among the most commonly prescribed classes of medications in Neonatal Intensive Care Units; however, its use has been constantly associated with a number of medication errors in clinical practice. In contrast to this situation, there is no common agreement when it comes to determining the right dosing, administration, or handling of antibiotics in this population. In order to help improve the use of antibiotics, decrease the rate of medication errors and optimize clinical results in the newborn, this review aims to provide recommendations to support and guide the correct preparation of some of the most relevant antibiotics used in neonatal wards.

Keywords: newborn; antimicrobials agents; dosing; drug administration; dilution.
\end{abstract}




\section{Introducción}

L os neonatos ingresados a Unidades de Cuidados Intensivos Neonatales (UCIN) están expuestos tempranamente en su vida a un gran número de medicamentos. Se estima que, en promedio, un recién nacido $(\mathrm{RN})$ en UCIN podría recibir aproximadamente cuatro cursos de medicamentos durante su hospitalización, pudiendo incluso aumentar hasta un promedio de 17 cursos en un RN de extremo bajo peso al nacer ${ }^{1}$. Al respecto, es sabido que el uso de antimicrobianos (ATM) concentra gran parte de ese número, siendo éstos uno de los grupos de fármacos más ampliamente utilizados en este tipo de pacientes ${ }^{1,2}$. A nivel nacional, Jiménez y cols., estimaron que alrededor de $32 \%$ de los RN hospitalizados estuvieron expuestos al menos a un $\mathrm{ATM}^{3}$; no obstante, este número puede variar dependiendo de las características y complejidad de la unidad neonatal evaluada ${ }^{4,5}$.

Si bien, en los últimos 10 años se han realizado considerables esfuerzos por mejorar los conocimientos en materia de dosificación, seguridad y efectividad de antimicrobianos en el $\mathrm{RN}^{6}$, la incidencia de errores asociados a su uso es aún elevada ${ }^{7-9}$, variando entre $38^{10}$ y $65 \%$ del total de medicamentos utilizados en algunos $\operatorname{casos}^{11}$. De estos errores, se ha observado por un lado que, el sobreuso de estos fármacos puede asociarse a un aumento en el riesgo de enterocolitis necrosante, candidiasis, sepsis neonatal tardía e incluso muerte ${ }^{8,9}$; y por otro, que la subdosificación estaría estrechamente relacionada a bajas concentraciones del ATM en el sitio de infección, pobre respuesta clínica y a un aumento en la probabilidad de selección de microorganismos multirresistentes ${ }^{12}$. Aun cuando lo anterior pudiese relacionarse más directamente con los objetivos de los Programas de Optimización de Uso de Antimicrobianos (PROA), estos problemas clínicos también podrían producirse como consecuencia de una inadecuada dosificación, preparación y/o administración de un ATM. Por consiguiente, resulta de vital importancia optimizar el uso y administración de este tipo de medicamentos en la población neonatal.

Los errores de administración son de los más comunes en $\mathrm{UCIN}^{13}$ pues en, parte, la compleja y cambiante farmacocinética del neonato desafía diariamente la necesidad de calcular y corregir los procesos de dosificación, dilución y manipulación de fármacos. Chappell y Newman documentaron que $31 \%$ de los medicamentos endovenosos prescritos en $\mathrm{RN}$, a dosis menores a un décimo de la dosis de presentación del vial (destinados para el uso primordialmente en adultos), resultaron en un aumento significativo de entre 10 y 100 veces en la incidencia de errores de administración ${ }^{14}$. Tomando estos puntos en consideración, y en vista del amplio uso de ATMs en el neonato, es especialmente importante concentrar los esfuerzos de todo el equipo multidisciplinario y propiciar la participación activa de médicos, farmacéuticos y enfermería/matronería para prevenir la ocurrencia de errores de este tipo. Así, con el objetivo de estandarizar la práctica clínica, y con ello disminuir las consecuencias asociadas al mal uso de estos medicamentos, esta revisión busca crear recomendaciones para la correcta manipulación y dilución de aquellos ATM de mayor relevancia en neonatología.

Las recomendaciones entregadas en la Tabla 1 fueron realizadas conforme a algunos de los ATM incluidos en el documento "Antimicrobianos en neonatología. Parte I: Recomendaciones de dosificaciones basadas en la más reciente evidencia en recién nacidos. Comité Consultivo de Infecciones Neonatales, Sociedad Chilena de Infectología"15.

A continuación, se discuten algunos de los aspectos más importantes relacionados al uso y preparación de ATMs en neonatología. Para ello, con el fin de unificar criterios y mejorar la comprensión de los conceptos desarrollados, la Tabla 2 resume algunos de los términos clave utilizados en esta revisión.

\section{Administración}

La falta de formulaciones farmacéuticas con dosis apropiadas para el neonato, con frecuencia, resulta en el uso off-label de medicamentos formulados para adultos ${ }^{37}$. Esta característica muchas veces obliga al profesional a manipular los medicamentos en múltiples ocasiones durante su preparación, aumentando en consecuencia las complicaciones asociadas a una dosis inadecuada ${ }^{38,39}$ (por ej.: subdosificación, sobredosificación, efectos adversos, etc.). Campino y cols., analizaron un total de 91 muestras, 52 de vancomicina y 39 de tobramicina, detectando errores de cálculo en 4,6\% y errores de precisión en 37,9\% de estos. Si bien los autores concluyeron que los errores registrados no resultaron en un perjuicio clínico evidente, sí reconocieron que estos son una fuente potencial de complicaciones graves, destacando la importancia de mejorar los métodos usados para la preparación de medicamentos de uso intravenoso ${ }^{40}$.

Por otro lado, los errores de dilución y/o reconstitución ocurridos durante la preparación de fármacos intravenosos pueden también significar un riesgo directo para el paciente neonatal ${ }^{41}$. En particular, la tolerancia de los vasos venosos puede verse directamente afectada por factores como la osmolaridad del medicamento. Así, el riesgo de infiltración o extravasación es mayor con el uso de soluciones consideradas hiperosmolares ( $>280 \mathrm{mOsm}$ en neonatos); no obstante, esta variable es posible de manejar con una reconstitución y/o dilución apropiada ${ }^{41}$. Como ejemplo de ello citamos el caso de ampicilina que, en comparación al proceso de reconstitución estándar con agua estéril para inyectables, al ser reconstituida en cloruro de sodio 9\%, aumenta hasta dos veces su osmo- 


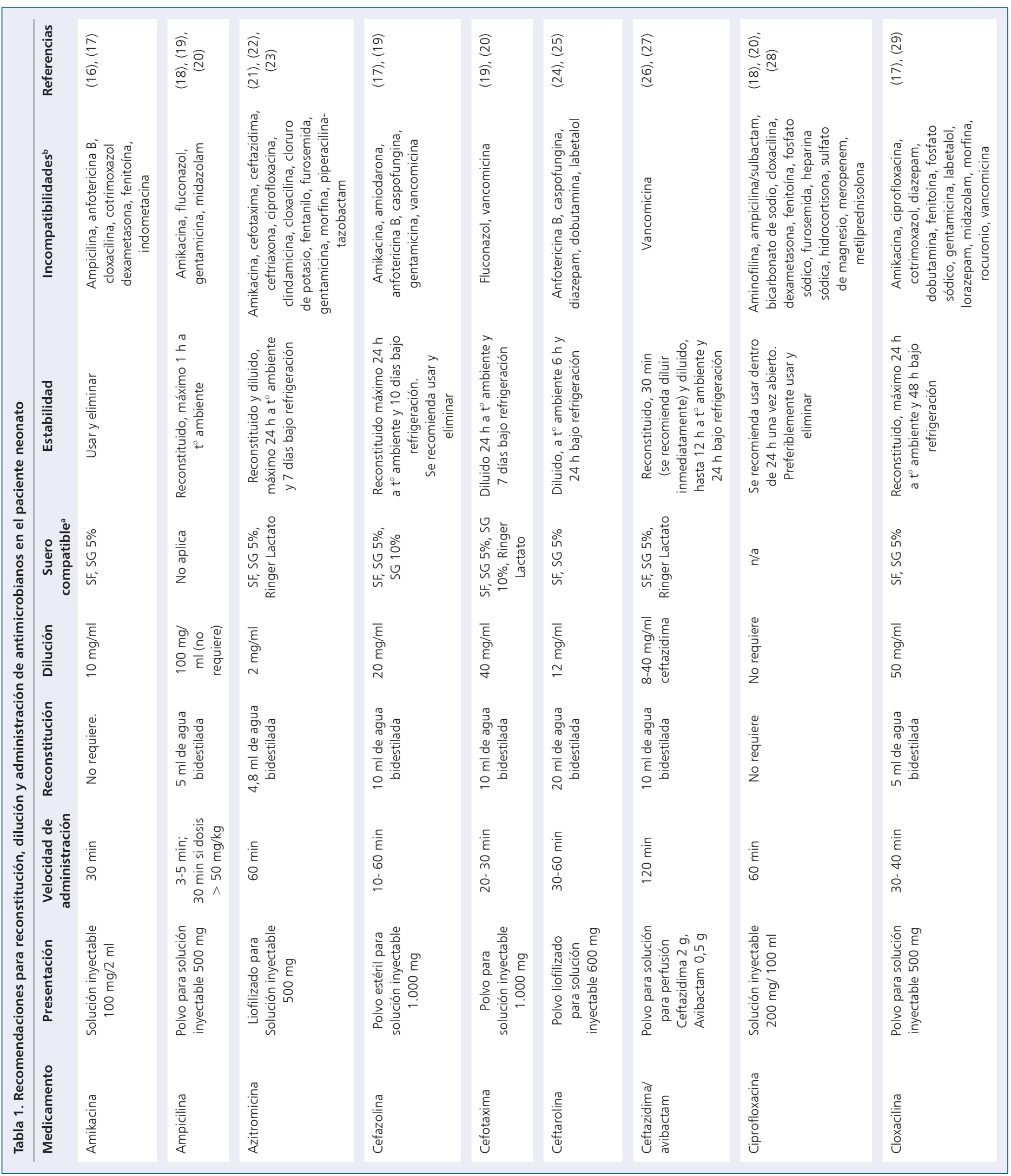




\begin{tabular}{|c|c|c|c|c|c|c|c|c|c|}
\hline 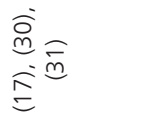 & $\underset{\mathbb{d}}{\stackrel{\mathbb{J}}{2}}$ & $\stackrel{\widehat{\infty}}{=}$ & $\widehat{\widetilde{N}}$ & 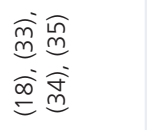 & $\underset{\mathbb{d}}{\overparen{\Im}}$ & $\begin{array}{l}\hat{o} \\
\tilde{m} \\
\hat{\tilde{m}} \\
\tilde{m}\end{array}$ & 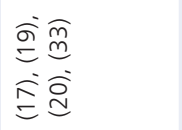 & $\underset{\mathbb{d}}{\stackrel{\mathbb{d}}{ }}$ & 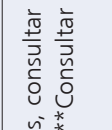 \\
\hline 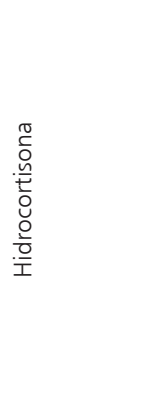 & 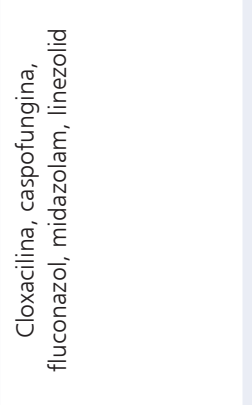 & 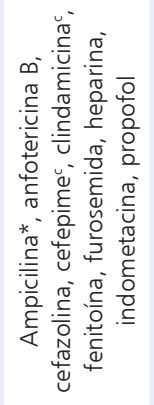 & 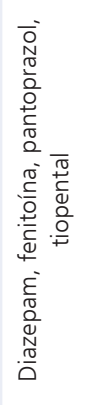 & 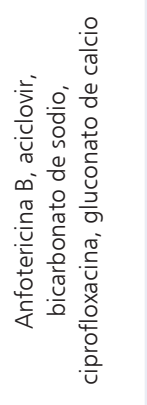 & 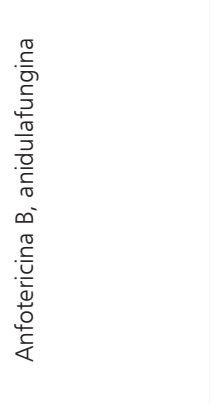 & 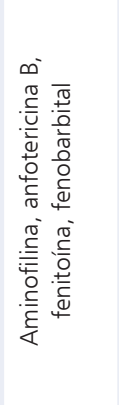 & 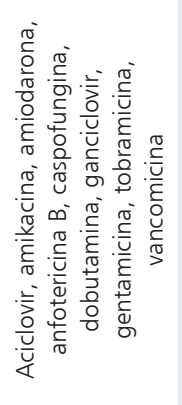 & 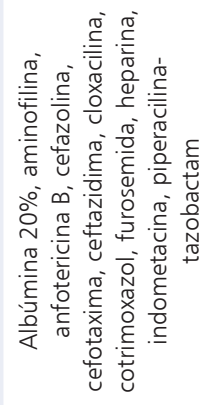 & 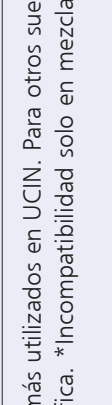 \\
\hline 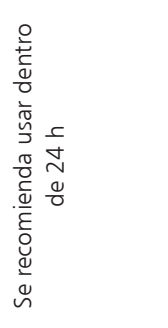 & 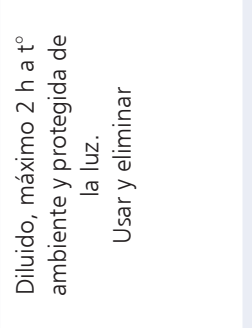 & 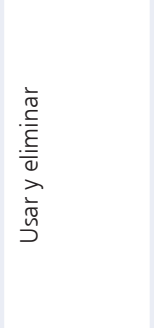 & 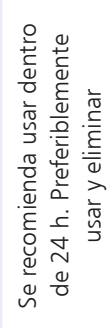 & 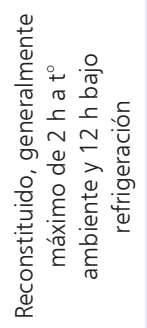 & 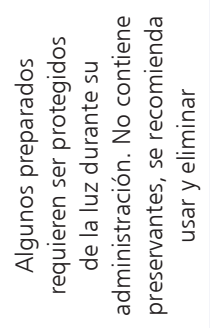 & 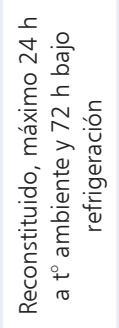 & 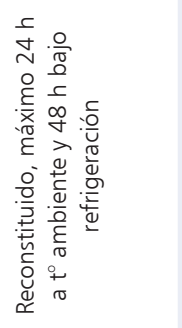 & 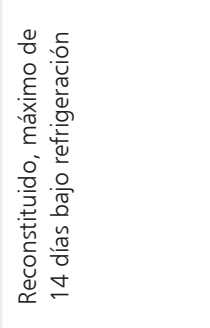 & 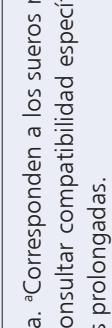 \\
\hline 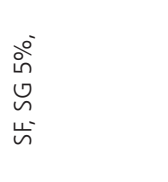 & $\begin{array}{l}\text { 吕 } \\
\text { 心 }\end{array}$ & $\begin{array}{l}\text { 今̊ } \\
\text { 心 } \\
\text { 出 }\end{array}$ & $\stackrel{\pi}{\Sigma}$ & 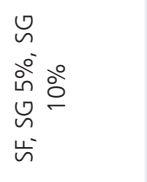 & $\stackrel{\pi}{\check{E}}$ & $\begin{array}{l}\text { 号 } \\
\text { Un } \\
\text { 岕 }\end{array}$ & 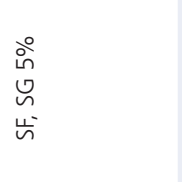 & 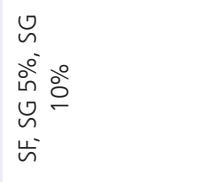 & 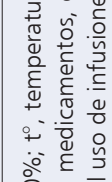 \\
\hline 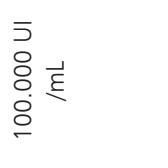 & 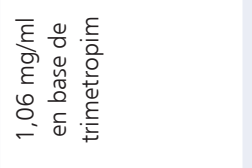 & $\begin{array}{l}\bar{\xi} \\
\stackrel{\xi}{\xi} \\
\stackrel{0}{-}\end{array}$ & 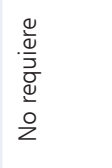 & 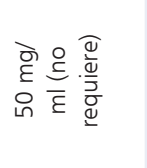 & 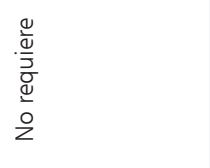 & $\begin{array}{l}\stackrel{8}{\xi} \bar{\xi} \\
\stackrel{\square}{\circ}\end{array}$ & 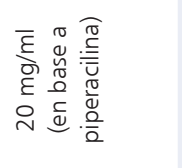 & 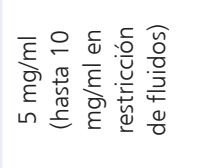 & 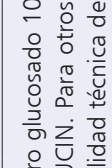 \\
\hline 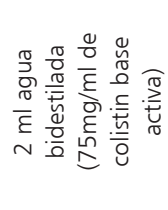 & $\begin{array}{l}\frac{0}{\mathscr{e}} \\
\frac{\vec{z}}{\bar{g}} \\
\frac{\mathbb{Q}}{2} \\
\frac{2}{2}\end{array}$ & 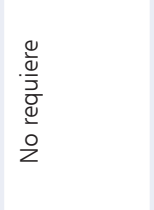 & 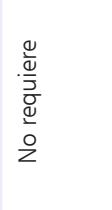 & 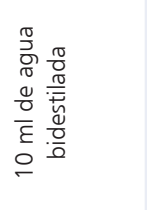 & 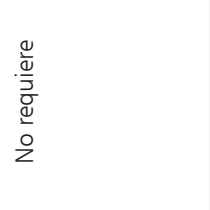 & 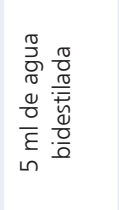 & 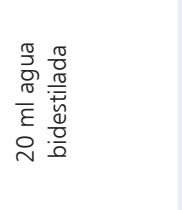 & 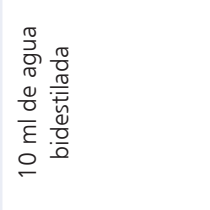 & 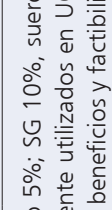 \\
\hline $\begin{array}{l}\stackrel{c}{\varepsilon} \\
\stackrel{\circ}{m}\end{array}$ & $\begin{array}{l}\frac{1}{\bar{\varepsilon}} \\
8 \\
\dot{0} \\
\dot{1}\end{array}$ & 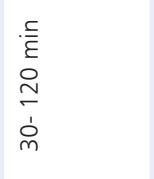 & 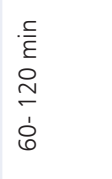 & 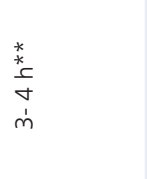 & 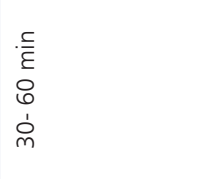 & $\begin{array}{l}\stackrel{c}{\bar{\varepsilon}} \\
0 \\
m \\
\stackrel{⺊}{\leftarrow}\end{array}$ & 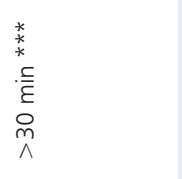 & $\begin{array}{l}\frac{c}{\varepsilon} \\
\stackrel{1}{\oplus} \\
\frac{1}{8}\end{array}$ & 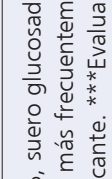 \\
\hline 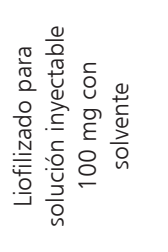 & 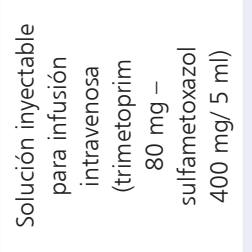 & 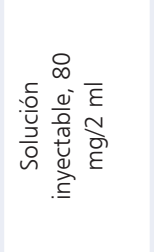 & 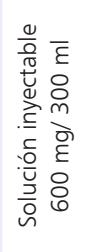 & 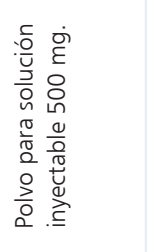 & 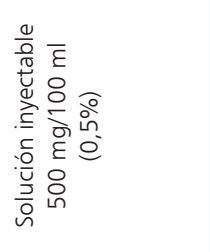 & 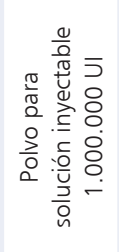 & 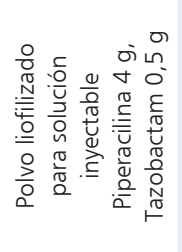 & 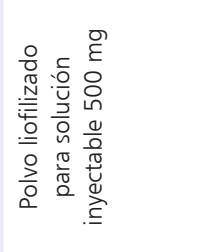 & 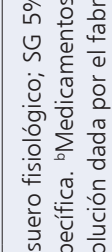 \\
\hline$\frac{c}{\frac{E}{\overline{0}}}$ & 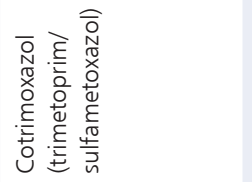 & 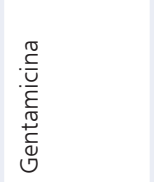 & 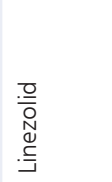 & 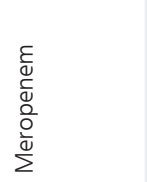 & 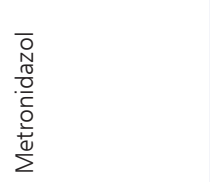 & 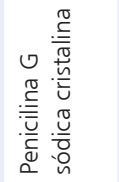 & 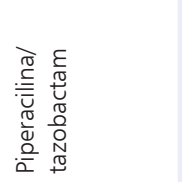 & 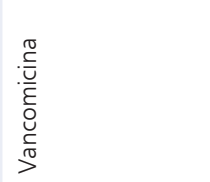 & 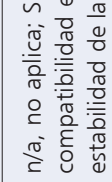 \\
\hline
\end{tabular}


laridad y, en consecuencia, también el potencial daño del territorio venoso y el tejido subcutáneo ${ }^{42}$.

Adicionalmente, otros factores del medicamento (como su pH y solubilidad) podrían tener el mismo efecto sobre el paciente ${ }^{41}$ de modo que, la recomendación, es siempre reconstituir y diluir -cuando sea necesario-procurando mantener concentraciones seguras e igualmente eficaces.

Para sobrellevar los problemas asociados a la individualización de las dosis calculadas ya sea según peso o edad postmenstrual, la Tabla 1 propone la preparación de distintos ATM endovenosos a concentraciones estandarizadas.

\section{Condiciones ambientales y de almacenamiento}

El efecto del ambiente sobre los ATMs es también un punto relevante a considerar. Las características fisiopatológicas y necesidades propias del paciente crítico neonatal condicionan un clima adverso para la estabilidad fisicoquímica de los medicamentos almacenados y administrados en una $\mathrm{UCIN}^{43,44}$ e imponen un límite de tolerancia térmica superior a la que algunos medicamentos pueden estar expuestos.

La estabilidad de los preparados farmacéuticos a temperatura ambiente es generalmente evaluada a temperaturas no mayores a $25^{\circ} \mathrm{C}^{45}$; sin embargo, la temperatura ambiente alcanzada en UCIN puede, en ocasiones, ser sustancialmente mayor ${ }^{46}$ afectando directamente las concentraciones in vivo de los medicamentos. Esto puede resultar en una disminución de la dosis de ATM expuesta al paciente, pudiendo eventualmente traducirse en un fracaso clínico o desarrollo de resistencia. Como ejemplo de ello, un estudio concluyó que más de $10 \%$ de meropenem es degradado luego de 12,8 y $6 \mathrm{~h}$ a temperaturas de $30,35 \mathrm{y} 40^{\circ} \mathrm{C}$, respectivamente, comprometiendo así la efectividad del tratamiento ${ }^{47}$.

Si bien la estabilidad de los ATM puede depender tanto de las características propias del medicamento como del tipo y calidad del proveedor, es siempre importante considerar las variaciones de temperatura y las condiciones de conservación para maximizar la entrega de tratamientos seguros y eficaces.

\section{Estabilidad en uso}

Los $\beta$-lactámicos son uno de los principales grupos de antimicrobianos cuya efectividad está determinada, farmacocinética y farmacodinámicamente, por el tiempo en que la concentración de fármaco libre se encuentra por sobre la concentración inhibitoria mínima (CIM) del microorganismo. En este contexto, el aumento de las concentraciones del fármaco en un período de tiempo acotado tendría efectos mínimos sobre el grado de destrucción bacteriana. Por el contrario, se ha demostrado que mantener una concentración de fármaco
Tabla 2. Definiciones

Reconstitución Adición de disolvente (generalmente agua para inyectables) al vial que contiene el medicamento en forma de polvo o liofilizado

Dilución $\quad$ Adición de diluyente (generalmente cloruro de sodio 0,9\% o suero glucosado $5 \%$ ) a una solución de medicamento con la finalidad de reducir su concentración inicial

Concentración Cantidad de medicamento que se encuentra disuelto en un volumen determinado de solución

Incompatibilidad Reacción ocurrida entre un medicamento endovenoso y su diluyente, contenedor $u$ otro medicamento endovenoso con el que esté en contacto ya sea en mezcla, en jeringa o sitio " $\mathrm{y}$ ". Esta reacción causa cambios visibles (ej. formación de precipitados, turbidez, cambios de color, etc.) o invisibles (ej. degradación del medicamento o de formación de material tóxico)

libre por encima de la CIM del microorganismo durante una parte del intervalo de dosificación predice de mejor manera la eficacia microbiológica ${ }^{48}$. Esto ha llevado a la implementación de regímenes de administración que consisten en la prolongación del tiempo de infusión o el uso de infusiones continuas de $24 \mathrm{~h}^{48}$. No obstante, este enfoque no está exento de complicaciones dado que, la estabilidad en uso de algunos antimicrobianos a distintas temperaturas, concentraciones y diluyentes podría influir negativamente sobre la degradación del fármaco o producción de compuestos tóxicos y llevar a una reducción en la efectividad, tal como se ha planteado para ceftazidima ${ }^{49}$. La Tabla 3 , muestra las estabilidades de algunos antimicrobianos a distintas temperaturas y concentraciones ${ }^{50}$.

Un ejemplo habitual en la práctica clínica es lo que ocurre con el uso de meropenem. Se ha demostrado que el uso de infusiones prolongadas de este fármaco aumenta la probabilidad de alcanzar parámetros farmacocinéticosfarmacodinámicos adecuados $\mathrm{y}$, por ende, beneficios clínicos a nivel de morbi-mortalidad, tanto en el paciente crítico adulto ${ }^{51}$ como en $\mathrm{RN}^{35}$. No obstante, en ocasiones resulta difícil ejecutar dichas indicaciones pues existe escaso consenso relacionado a la estabilidad en solución de meropenem. Por un lado, Fawaz y cols., proponen que, a concentraciones de $14,3 \mathrm{mg} / \mathrm{mL}$, a temperatura de $21^{\circ} \mathrm{C}$, el medicamento sería estable por un período menor o igual a $7 \mathrm{~h}^{52}$ y por otro, Venugopalan y cols., respaldan el uso de mezclas de $10 \mathrm{mg} / \mathrm{mL}$ diluidas en solución salina fisiológica $(\mathrm{NaCl}$ 9\%) para infusiones menores o iguales a $12 \mathrm{~h}^{53}$. De esta forma, y ante la disparidad de información disponible tanto en la literatura como en el mercado, la recomendación es siempre consultar los datos de estabilidad entregados por el proveedor y, con ello, optimizar la entrega de la terapia antimicrobiana. 


\begin{tabular}{|c|c|c|c|c|c|c|}
\hline Fármacos & $\mathrm{T}^{\circ}\left(\mathrm{C}^{\circ}\right)$ & Concentración & Solución & $\begin{array}{c}\text { Estabilidad } \\
\text { (horas) }\end{array}$ & Recomendación & Observación \\
\hline \multirow[t]{3}{*}{ Ampicilina/sulbactam } & 37 & $\leq 30 / 15 \mathrm{mg} / \mathrm{ml}$ & SF & 24 & \multirow[t]{3}{*}{ Infusión de 4 h } & \\
\hline & 25 & $\leq 30 / 15 \mathrm{mg} / \mathrm{ml}$ & SF & 8 & & \\
\hline & 4 & $\leq 30 / 15 \mathrm{mg} / \mathrm{ml}$ & SF & 48 & & \\
\hline \multirow[t]{3}{*}{ Cefepime } & 37 & $\leq 40 \mathrm{mg} / \mathrm{ml}$ & SF & 8 & \multirow[t]{3}{*}{ Infusión 24 h } & \\
\hline & 25 & $\leq 40 \mathrm{mg} / \mathrm{ml}$ & SF & 24 & & \\
\hline & 4 & $\leq 40 \mathrm{mg} / \mathrm{ml}$ & SF & $\geq 24$ & & \\
\hline \multirow[t]{3}{*}{ Ceftazidima } & 37 & $<3 \mathrm{~g} / 100 \mathrm{~mL}$ & SF & 8 & \multirow[t]{3}{*}{ Infusión 24 h } & \multirow{3}{*}{$\begin{array}{l}\text { Pirimidina, subproducto de degradación de ceftazidima, } \\
\text { es un problema de toxicidad teórica se minimiza limitan- } \\
\text { do máxima dosis día } 6 \mathrm{~g} \text { a }<3 \mathrm{~g} / 100 \mathrm{~mL} \text { (SF) }\end{array}$} \\
\hline & 25 & $<3 \mathrm{~g} / 100 \mathrm{~mL}$ & SF & 24 & & \\
\hline & 4 & $<3 \mathrm{~g} / 100 \mathrm{~mL}$ & SF & $\geq 24$ & & \\
\hline \multirow[t]{3}{*}{ Meropenem } & 37 & $1-20 \mathrm{mg} / \mathrm{mL}$ & SF & $<4$ & \multirow[t]{3}{*}{ Infusión de 3 h } & \\
\hline & 25 & $1-20 \mathrm{mg} / \mathrm{mL}$ & SF & 4 & & \\
\hline & 4 & $1-20 \mathrm{mg} / \mathrm{mL}$ & SF & 24 & & \\
\hline \multirow[t]{3}{*}{ Piepracilina/tazobactam } & 37 & $20-80 \mathrm{mg} / \mathrm{mL}$ & SF & 24 & \multirow[t]{3}{*}{ Infusión de 4 h } & \\
\hline & 25 & $20-80 \mathrm{mg} / \mathrm{mL}$ & SF & 24 & & \\
\hline & 4 & $20-80 \mathrm{mg} / \mathrm{mL}$ & SF & ND & & \\
\hline
\end{tabular}

\section{Incompatibilidades}

Dado el limitado número de accesos venosos en el paciente neonatal, a menudo es necesario administrar varios medicamentos a través de una misma vía. Así, es importante considerar el potencial de incompatibilidades (tanto físicas como químicas) entre los distintos medicamentos, sus solventes y los materiales utilizados para su administración. Las incompatibilidades intravenosas pueden alterar las propiedades de uno o más de los fármacos involucrados, resultando, muchas veces, en pérdidas significativas de la actividad terapéutica y/o una

Tabla 4. Consecuencias clínicas de las incompatibilidades intravenosas por mezcla de fármacos

Pérdida de actividad de los principios activos involucrados

Reacciones adversas inespecíficas por generación de productos tóxicos

Obstrucción de la vía por donde se administra

Formación de precipitados con riesgos de trombosis pulmonar, granulomas locales, etc.

Reducción del flujo de la vía (independiente de la precipitación)

Inflamación local, incluso flebitis química exposición innecesaria a agentes potencialmente nocivos para el paciente (Tabla 4) ${ }^{54}$. De este modo, es siempre recomendable evaluar la aparición de fenómenos de este tipo ya sean visibles (por ej.: formación de precipitados, turbidez, cambios de color, cambios en la viscosidad o formación de gas) o invisibles (por ej.: reacciones químicas de degradación o de formación de material tóxico). En relación al uso de ATMs, este tema adquiere particular importancia pues, en el ambiente de UCIN, tanto ampicilina como gentamicina han sido descritos como los dos medicamentos con mayor potencial de experimentar incompatibilidades con otros fármacos ${ }^{55}$ (Tabla 1) y, por ende, distintas estrategias debiesen ser aplicadas para evitar la administración simultánea de estos antimicrobianos con otros medicamentos.

Las incompatibilidades en las UCIN pueden ser extremadamente peligrosas, con un impacto clínico significativo, e incluso, asociadas a consecuencias fatale ${ }^{56}$ pues, a diferencia de los adultos, el $\mathrm{RN}$ presenta riesgos adicionales. Factores como la necesidad de restringir el aporte de volumen, tiempos de infusión más prolongados, la disponibilidad de capilares pequeños y la pobre integridad del territorio venoso facilitarían la obstrucción por precipitados y aumentarían la gravedad del evento ${ }^{55,56}$. Un ejemplo de lo anterior es lo ocurrido con ceftriaxona, antimicrobiano actualmente no utilizado en unidades neonatales pues se logró demostrar que, al utilizarse 
equipos clínicos a incurrir en prácticas que no necesariamente aseguran la entrega de un tratamiento seguro y eficaz. De esta forma, y para optimizar la terapia antimicrobiana en estos pacientes, factores como las condiciones de almacenamiento, la velocidad de administración, la estabilidad en solución y las incompatibilidades necesitan siempre ser consideradas.

Por último, la implementación de medidas como la estandarización de las concentraciones de ATM utilizadas o la disponibilidad de centrales de mezclas intravenosas centralizadas, asociadas a un equipo multidisciplinario que participe en la adjudicación de licitaciones (con el fin de verificar todos los detalles ya mencionados) pueden contribuir a disminuir los errores de medicación y mejorar la calidad de los ATMs utilizados en nuestros pacientes.

Este documento propone una pauta actualizada y específica para el uso de ATMs en neonatología, basada en la evidencia disponible y considerando, tanto aspectos relativos a las características del $\mathrm{RN}$, como también las características propias del medicamento, todo esto con el fin de servir de guía para la manipulación de medicamentos, sin desmedro de aquellas recomendaciones desarrolladas de forma independiente por cada centro asistencial. Con ello, y por medio de la estandarización de la práctica clínica, se espera contribuir a minimizar los riesgos asociados al uso de antimicrobianos en esta población, mejorar los resultados clínicos y garantizar la entrega de tratamientos seguros y eficaces al paciente neonatal.

Agradecimientos. A los miembros del Comité Consultivo de Infecciones Neonatales de la Sociedad Chilena de Infectología, por su contribución a la revisión de este documento.

\section{Referencias bibliográficas}

1.- Hsieh E M, Hornik C P, Clark R H, Laughon M M, Benjamin D K. Jr, Smith P.B. Medication use in the neonatal intensive care unit. Am. J. Perinatol. 2014; 31: 811-22. doi: 10.1055/s0033-1361933.

2.- Clark R H, Bloom B T, Spitzer A R, Gerstmann D R. Reported medication use in the neonatal intensive care unit: date from a large national data set. Pediatrics 2006; 117: 1979-87. doi: 10.1542/peds.2005-1707.

3.- Jiménez E, Valls N, Astudillo P, Valls C, Cavada G, Sandoval A, et al. Evaluación del consumo de antimicrobianos en una unidad de neonatología: un trabajo en equipo para promover el uso racional de antimicrobianos. Rev Chilena Infectol 2017; 34 (6): 544-52. doi: 10.4067/S0716-10182017000600544.

4.- Cantey J B, Wozniak P S, Sánchez P J. Prospective surveillance of antibiotic use in the neonatal intensive care unit: results from the SCOUT study. Pediatr Infect Dis J 2015; 34: 267-72. doi: 10.1097/ INF.0000000000000542.

5.- Schulman J, Benitz W E, Profit J, Lee H, Dueñas G, Bennett M V, et al. Newborn antibiotic exposures and association with proven bloodstream infection. Pediatrics 2019;144 (5): e20191105. doi: 10.1542/ peds.2019-1105.

6.- Johnson J K, Laughon M M. Antimicrobial agent dosing in infants. Clin Ther 2016; 38 (9): 1948-60. doi: 10.1016/j.clinthera.2016.06.017.

7.- Van Donge T, Bielicki J.A, van den Anker J, Pfister M. Key components for antibiotic dose optimization of sepsis in neonates and infants. Front. Pediatr 2018; 6: 325. doi: 10.3389/fped.2018.00325. doi: 10.3389/ fped.2018.00325.

8.- Tripathi N, Cotten C M, Smith P B. Antibiotic use and misuse in the neonatal intensive care unit. Clin Perinatol 2012; 39 (1): 61-8. doi: 10.1016/j.clp.2011.12.003.

9.- Cotton C M. Adverse consequences of neonatal antibiotic exposure. Curr Opin Pediatr 2016; 28 (2): 141-9. doi: 10.1097/ MOP.0000000000000338.

10.- Pawluk S, Jaam M, Hazi F, Al Hail M S, El Kassem W, Khalifa H, et al. A description of medication errors reported by pharmacists in a neonatal intensive care unit. Int J Clin Pharm 2017;39 (1): 88-94. doi: 10.1007/s11096-0160399-x.

11.- Eslami K, Aletayeb F, Aletayeb S, Kouti L, Hardani A. Identifying medication errors in neonatal intensive care units: a two-center study. BMC Pediatrics 2019; 19 (1): 365. doi: 10.1186/s12887-019-1748-4.

12.- Patel S J, Saiman L. antibiotic resistance in neonatal intensive care unit pathogens: mechanisms, clinical impact, and prevention including antibiotic stewardship. Clin 
Perinatol 2010; 37: 547-63. doi: 10.1016/j. clp.2010.06.004.

13.- Krzyzaniak N, Bajorek B. Medication safety in neonatal care: a review of medication errors among neonates. Ther Adv Drug Saf 2016; 7 : 102-19. doi: 10.1177/2042098616642231.

14.- Chappell K, Newman C. Potential tenfold drug overdoses on a neonatal unit. Arch Dis Childhood Fetal Neonatal 2004; 89: F483-F484. doi: 10.1136/adc.2003.041608.

15.- Sandoval C A, Aravena U M, Cofré S F, Delpiano M L, Hernández M R, Hernández E M, et al. Antimicrobianos en neonatología. Parte I: Recomendaciones de dosificaciones basadas, en la más reciente evidencia en recién nacidos Comité Consultivo de Infecciones Neonatales, Sociedad Chilena de Infectología. Rev Chilena Infectol 2020; 37 (5): 490-508. http://dx.doi.org/10.4067/S071610182020000500490 .

16.- Murray K L, Wright D, Laxton B, Miller K $\mathrm{M}$, Meyers J, Englebright J. Implementation of standardized pediatric i.v. medication concentrations. Am J Health-Syst Pharm 2014; 71(17): 1500-8. doi: 10.2146/ ajhp140024.

17.- Lexicomp Online, Pediatric and Neonatal Lexi-Drugs Online, Hudson, Ohio: UpToDate, Inc.2013 [citado el 29 de julio de 2020].

18.- Lee K, Hagemann T, Thompson A J, Phelps S. Pediatric Injectable Drugs: The Teddy Bear Book (Pediatric Injectable Drugs). $10^{\circ}$ ed. Bethesda (Maryland): American Society of Health-System Pharmacists; 2013.

19.- Truven Health Analytics. NeoFax [Internet]. Greenwood Village (CO): Truven Health Analytics; 2020. Disponible en: https://neofax. micromedexsolutions.com/ [citado el 29 de julio de 2020].

20.- British National Formulary for Children (Versión 3.0.8 (854)) [Mobile application software]. Google Play Store. Disponible en: https://play.google.com/ store/apps/details?id=com.medhand. bnfc2019x1\&hl=es_419\&gl=US.

21.- Zitromax (Azitromicina) [folleto de información para profesionales]. Pfizer; noviembre, 2015. https://www.pfizerpro. com.co/sites/pfizerpro.com.co/files/ g10059421/f/201903/lld_azitromicina_ Zitromax_IV_cds12.pdf.

22.- Viscardi R, Terrin M, Magder L, Davis N, Dulkerian S, Waites K, et al. Randomised trial of azithromycin to eradicate Ureaplasma in preterm infants. Arch Dis Child Fetal Neonatal Ed 2020; 105 (6): 615-22. doi: 10.1136/ archdischild-2019-318122.

23.- Viscardi R, Othman A, Hassan H, Eddington $\mathrm{N}$, Abebe E, Terrin M, Kaufman D, et al. Azithromycin to prevent bronchopulmonary dysplasia in ureaplasma-infected preterm infants: pharmacokinetics, safety, microbial response, and clinical outcomes with a 20-milligram-per-kilogram single intravenous dose. Antimicrob Agents Chemother 2013; 57(5): 2127-33. doi: 10.1128/AAC.02183-12.

24.- Trissel L. The Handbook of Injectable Drugs. $19^{\circ}$ ed. Bethesda (Maryland): American Society of Health-System Pharmacists; 2016.

25.- Zinforo ${ }^{\circledR}$ (ceftarolina fosamil) [folleto de información para profesionales]Pfizer; agosto 2016. Disponible en: https://www. pfizerpro.cl/sites/g/files/g10049911/f/201808/ ZINFORO\%20POLVO\%20PARA\%20 SOL. $\% 20$ CONCENTRADA $\% 20$ PARA $\% 20$ INF.\%20600\%20mg\%20-\%20Jul\%202018.pdf

26.- Zaficefta ${ }^{\circledR}$ (ceftazidima pentahidrato y avibactam sódico) [folleto de información para profesionales]. Pfizer; Enero, 2017.

27.- Avycaz $^{\mathbb{B}}$ (ceftazidime/avibactam) [folleto de información para profesionales]. Irvine, CA: Allergan USA Inc; October 2019.

28.- Pacifici G M, Marchini G. Clinical pharmacology of ciprofloxacin in neonates: Effects and pharmacokinetics. Int J Pediatr 2017; 5(6): 5023-41. https://dx.doi. org/10.22038/ijp.2017.23193.1952.

29.- Jimeno S, Ortiz R, Riaza M. Guía Farmacoterapéutica Neonatal. SegurneoSen. 2019. Disponible en: https:// mariamontanavivas.wordpress.com/2020/01/14/ guia-farmacoterapeutica-neonatal-nuevaedicion-gratuita-y-revisada-2019/ [citado el 22 de febrero de 2021].

30.- Jajoo M, Kumar V, Jain M, Kumari $\mathrm{S}$, Manchanda, V. Intravenous colistin administration in neonates. Pediatr Infect Dis J 2011; 30 (3): 218-21. doi: 10.1097/ INF.0b013e3182064bfe.

31.- Labuschagne Q, Schellack N, Gous A, Bronkhorst E, Schellack G, van Tonder L, et al. COLISTIN: adult and paediatric guideline for South Africa, 2016. S Afr J Infec Dis 2016; 31(1): 3-7. doi: $10.1080 / 23120053.2016 .1144285$

32.- Zhang Y, Trissel L A. Compatibility of linezolid injection with intravenous administration sets. J Am Pharm Assoc (Wash) 2001; 41: 285-6. doi: 10.1016/s10865802(16)31237-2.

33.- Ainsworth S. Neonatal Formulary 7: Drug use in pregnancy and the first year of life. $7^{\circ} \mathrm{Ed}$. Chichester (West Sussex), John Wiley \& Sons Inc.; 2015.

34.- Padari H, Metsvaht T, Kõrgvee L, Germovsek E, Ilmoja M, Kipper K, Herodes K, et al. Short versus long infusion of meropenem in verylow-birth-weight neonates. Antimicrob Agents Chemother 2012; 56(9): 4760-4. doi: 10.1128/ AAC.00655-12.

35.- Shabaan A E, Nour I, Eldegla H E, Nasef N, Shouman B, Abdel-Hady H. Conventional versus prolonged infusion of meropenem in neonates with gram-negative late-onset sepsis.
Pediatr. Infect. Dis. J. 2017; 36: 358-63. doi: 10.1097/INF.0000000000001445.

36.- Stiles M L, Tu Y H, Allen L V Jr. Stability of cefazolin sodium, cefoxitin sodium, ceftazidime, and penicillin $\mathrm{G}$ sodium in portable pump reservoirs. Am J Hosp Pharm 1989; 46: 1408-12. PMID: 2667354

37.- Cuzzolin L. Off-label drug in the newborn. J Pediatr Neonat Individual Med 2014; 3; 2 : 1-8. https://jpnim.com/index.php/jpnim/article/ view/030224/182.

38.- Arenas-López S, Stanley I M, Tunstell P, Aguado-Lorenzo V, Philip J, Perkins J, et al. Safe implementation of standard concentration infusions in paediatric intensive care. J Pharm Pharmacol 2017; 69: 529-36.

39.- Kaushal R, Bates D W, Landrigan C, McKenna K J, Clapp M D, Federico F, et al. Medication errors and adverse drug events in pediatric inpatients. JAMA 2001; 285: $2114-$ 20. doi: 10.1001/jama.285.16.2114.

40.- Campino E, Santesteban M, García M, Rueda A, Valls-i-Soler. Errores en la preparación de fármacos intravenosos en una Unidad de Cuidados Intensivos Neonatal. Una potencial fuente de eventos adversos. An Pediatr (Barc) 2013; 79: 21-5. doi: 10.1016/j. anpedi.2012.09.005

41.- Beall V, Hall B, Mulholland J, Gephart S. Neonatal extravasation: an overview and algorithm for evidence-based treatment. Newborn Infant Nurs Rev 2013; 13: 189- 95. https://doi.org/10.1053/j.nainr.2013.09.001.

42.- Pettit J. Assessment of the infant with a peripheral intravenous device. Adv Neonatal Care 2003; 3: 230-40. PMID: 14648520.

43.- Allegaert K, van den Anker J. Neonatal drug therapy: The first frontier of therapeutics for children. Clin Pharmacol Ther 2015; 98: 28897. doi: 10.1002/cpt.166.

44.- White R, Smith J, Shepley M. Recommended standards for newborn ICU design, eighth edition. J Perinatol 2013; 33(S1): S2-S16. doi: 10.1038/jp.2013.10.

45.- World Health Organization Expert Committee on Specifications for Pharmaceutical Preparations. Directrices para las pruebas de estabilidad de productos farmacéuticos que contienen sustancias medicamentosas bien establecidas en formas farmacéuticas corrientes. Geneva: World Health Organization; 2006. https://www.paho.org/ hq/dmdocuments/2008/5_Anexo_5_del informe_34.pdf.

46.- Thomas K A, Magbalot A, Shinabarger K, Mokhnach L, Anderson M, Diercks K, et al. Seasonal mapping of NICU temperature. Adv Neonatal Care 2010; 10(2): 83-7. https://doi. org/10.1097/anc.0b013e3181d50d31.

47.- Keel R, Sutherland C, Crandon J, Nicolau D. Stability of doripenem, imipenem and meropenem at elevated 
room temperatures. Int. J. Antimicrob Agents 2011; 37(2): 184-5. doi: 10.1016/j. ijantimicag.2010.06.043

48.- Onufrak N J, Forrest A, González D. Pharmacokinetic and pharmacodynamic principles of anti-infective dosing. Clin Ther 2016; 38 (9): 1930-47. doi: 10.1016/j. clinthera.2016.06.015.

49.- Jones T E, Selby P R, Mellor C S, Cheam D B. Ceftazidime stability and pyridine toxicity during continuous i.v. infusion. Am J Health Syst Pharm 2019; 76 (4): 200-5. doi: 10.1093/ ajhp/zxy035. PMID: 30715181.

50.- "The Sanford Guide to Antimicrobial Therapy. Dallas, TX: Antimicrobial Therapy, Inc. Continuous/Prolonged Infusion Dosing. Sandford Guide online, Updated Jan 22, 2021.

51.- Yu Z, Pang X, Wu X, Shan C, Jiang S. Clinical outcomes of prolonged infusion (extended infusion or continuous infusion) versus intermittent bolus of meropenem in severe infection: a meta-analysis. PLoS One 2018;13(7): e0201667. doi: 10.1371/journal. pone. 0201667 .

52.- Fawaz S, Barton S, Whitney L, Swinden J,
Nabhani-Gebara S. Stability of meropenem after reconstitution for administration by prolonged infusion. Hosp Pharm 2019; 54(3): 190-6. doi: 10.1177/0018578718779009

53.- Venugopalan V, Manigaba K, Borgert S J, Cope J, Peloquin C A, Klinker K P. Training a drug to do new tricks: insights on stability of meropenem administered as a continuous infusion. Microbiol Insights 2018; 11: 1178636118804549. doi:10.1177/1178636118804549.

54.- Bertsche T, Mayer Y, Stahl R, HoppeTichy T, Encke J, Haefeli W. E. Prevention of intravenous drug incompatibilities in an intensive care unit. Am J Health-Syst Pharm 2008; 65(19): 1834-40. doi: 10.2146/ ajhp070633.

55.- Leopoldino R W, Costa H T, Costa T X, Martins R R, Oliveira A G. Potential drug incompatibilities in the neonatal intensive care unit: a network analysis approach. BMC Pharmacol Toxicol 2018; 19: 83. https://doi. org/10.1186/s40360-018-0265-7.

56.- De Giorgi I, Guignard B, Fonzo-Christe C, Bonnabry, P. Evaluation of tools to prevent drug incompatibilities in paediatric and neonatal intensive care units. Pharm World Sci 2010; 32(4): 520-9. doi: 10.1007/s11096-0109403-z.

57.- Bradley J, Wassel R, Lee L, Nambiar S. Intravenous ceftriaxone and calcium in the neonate: assessing the risk for cardiopulmonary adverse events. Pediatrics 2009; 123(4): e609-e613. doi: 10.1542/peds.2008-3080.

58.- Acantex ${ }^{\circledR}$ (ceftriaxona) [folleto de información para profesionales]. Roche; julio, 2016. https:// www.roche.cl/content/dam/rochexx/roche-cl/ roche_chile/es_CL/corporate/Files/Folleto $\% 20$ Medico\%20Acantex\%20\%20CDS\%2060\%20 $\% 20 H A \% 20$ request $\% 20 j u l i o \% 202014$.pdf.

59.- Robinson S, Harris A, Atkinson S, Atterbury C, Bolton-Maggs P, Elliott C, et al. Standardization of infusion solutions to reduce the risk of incompatibility. Am J Health-Syst Pharm 2008; 65 (17): 1648-54. doi: 10.2146/ ajhp070471.

60.- Perez M, Décaudin B, Abou Chahla W, Nelken B, Storme L, Masse M, et al. Effectiveness of in-line filters to completely remove particulate contamination during a pediatric multidrug infusion protocol. Sci Rep 2018; 8: 7714. https://doi.org/10.1038/s41598-018-25602-6. 